\title{
Diabetogenesis in
} Rheumatoid Arthritis: Do Inflammation and Glucocorticoid-induced
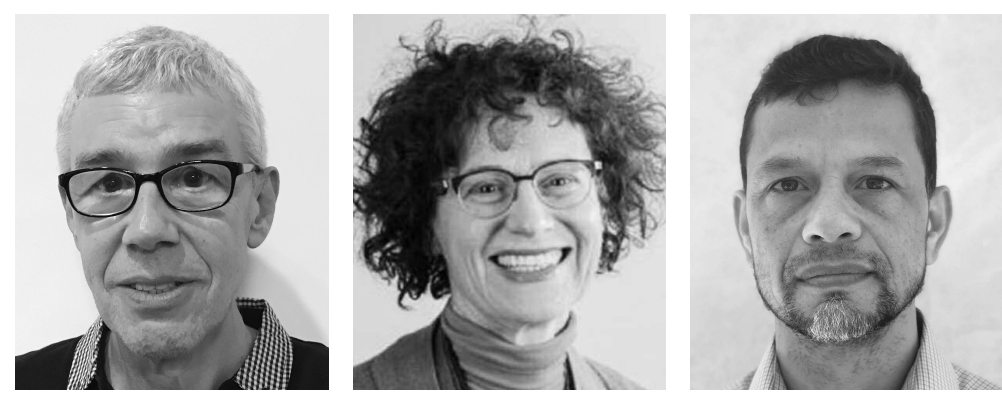

\section{Incretin Overproduction Cause $\beta$-Cell \\ Overcompensation and Consequent Premature Decompensation?}

Metabolic abnormalities occur frequently in rheumatoid arthritis (RA) ${ }^{1}$. In this regard, metaanalyses documented an enhanced diabetes prevalence (OR 1.74, 95\% CI 1.22-2.50) in $\mathrm{RA}^{2}$. Diabetes increases the risk of combined cardiovascular (CV) morbidity including myocardial infarction, angina, heart failure, stroke, and peripheral artery disease (RR $1.94,95 \%$ CI $1.58-2.30$ ) in $\mathrm{RA}^{3}$. Poor RA activity control strongly predicts incident-impaired fasting glucose and type 2 diabetes ${ }^{4}$. Diabetes is likely to account for a substantial proportion of the enhanced CV disease burden in RA.

In healthy persons, glucose homeostasis is maintained by optimal (1) insulin sensitivity; (2) hepatic insulin extraction; (3) pancreatic $\beta$-cell insulin secretion; and (4) interactions of all three ${ }^{5}$. Type 2 diabetes development typically comprises the sequential stages of long-lasting adverse lifestyle factor (excess adiposity and physical inactivity)-induced insulin resistance and reduced hepatic insulin extraction that exacerbates reduced insulin sensitivity, compensatory increased $\beta$-cell insulin secretion, and $\beta$-cell decompensation with reduced insulin secretion that results in prediabetes [impaired fasting glucose (5.6-6.9 mmol/l), impaired glucose tolerance (7.8-11.0 mmol/1), and/or raised hemoglobin A1c concentrations $(6.0-6.4 \% / 42-46 \mathrm{mmol} / \mathrm{l})]$ and ultimately diabetes (Figure 1).

Insulin resistance is a pathological state in which the ability of insulin to transport glucose into the cells is impaired. It associates with other metabolic abnormalities and increases $\mathrm{CV}$ risk, but in itself does not cause diabetes ${ }^{6,7,8}$. By contrast, impaired $\beta$-cell function is essential in diabetes development and is genetically mediated ${ }^{7,8}$. Whereas genetic factors are also implicated in impaired hepatic insulin extraction, its main determinant is pulsatile pancreatic insulin secretion extent (insulin release pulse mass) into the portal vein ${ }^{8}$. Increased free fatty acid concentrations and steatosis are additional causes of impaired hepatic insulin extraction ${ }^{5}$.

Methods used to determine insulin sensitivity and/or $\beta$-cell function include the homeostasis model assessment (HOMA and HOMA2), frequently sampled oral glucose tolerance tests (OGTT) and intravenous glucose tolerance tests, hyperglycemic and euglycemic-hyperinsulinemic clamp procedures, and meal tolerance tests ${ }^{9}$. Insulin and equimolar co-secreted C-peptide production occurs in a reciprocal and proportional response to insulin resistance in an attempt to maintain glucose homeostasis ${ }^{9}$. Therefore, insulin sensitivity needs to be accounted for when estimating $\beta$-cell function. This can be effectively achieved by determining the disposition index, which is a measure of insulin secretion during the prevailing degree of insulin action. The disposition index can be calculated as the product between insulin sensitivity and insulin production and is a constant for a given degree of glucose tolerance ${ }^{7}$. Accordingly, the disposition index is reduced in diabetes and prediabetic conditions including impaired fasting glucose and glucose tolerance, a family history of type 2 diabetes, polycystic ovarian syndrome, former gestational diabetes, and advanced age. Forty to $80 \%$ of secreted insulin is cleared by the liver. By contrast, C-peptide is not metabolized by the liver ${ }^{8}$. Therefore, the molar ratio of circulating C-peptide to insulin represents a surrogate marker of hepatic insulin extraction.

Less is known about diabetogenesis in RA. Increased cytokine production (a prominent feature of RA) can impair both insulin sensitivity and $\beta$-cell function ${ }^{6,10}$. Insulin

See Insulin sensitivity in RA, page 229

Personal non-commercial use only. The Journal of Rheumatology Copyright @ 2019 . All rights reserved. 


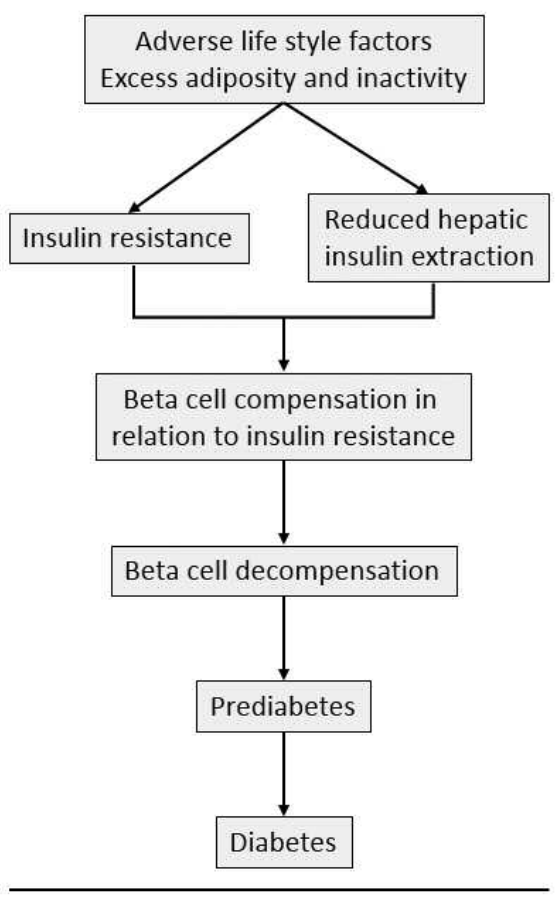

Non-RA subjects

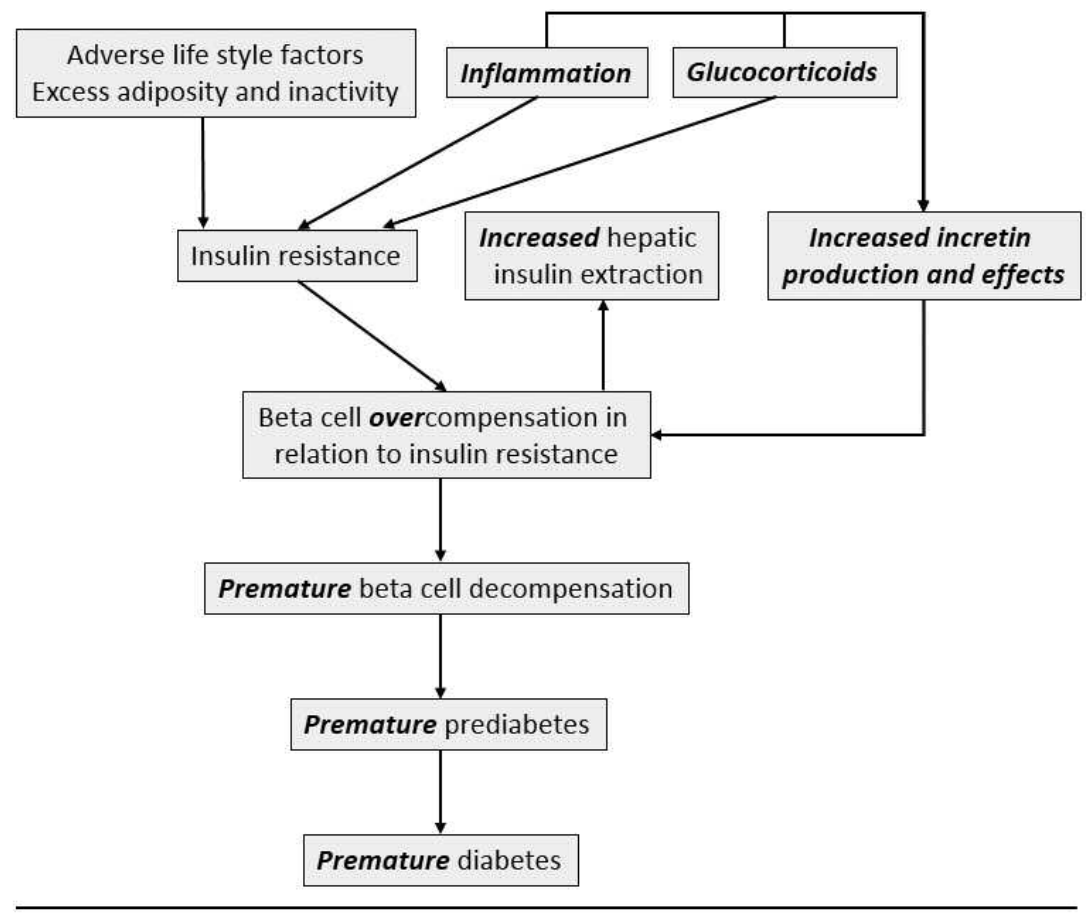

Patients with RA

Figure 1. Proposed sequential diabetogenesis stages in patients with RA compared to those without. The main differences are shown in bold italics. RA: rheumatoid arthritis.

resistance has been amply reported in $\mathrm{RA}^{10}$. Characteristics that associate with impaired insulin sensitivity in RA include not only excess adiposity but also systemic inflammation, chronic oral glucocorticoid therapy, and subclinical hypothyroidism ${ }^{6,10,11,12}$.

Ferraz-Amaro and colleagues ${ }^{13}$ found that patients with RA experience increased proinsulin production, which represents impaired insulin processing and $\beta$-cell function. Disease activity relates to impaired $\beta$-cell function as determined by HOMA $\beta$-cell function in $\mathrm{RA}^{11}$. This relationship is independent of insulin resistance risk factors ${ }^{11}$. Both synthetic conventional and biological disease-modifying antirheumatic drugs (DMARD) increase insulin sensitivity in $\mathrm{RA}^{6,10,14,15}$. Tumor necrosis factor- $\alpha$ inhibition ${ }^{14}$ and hydroxychloroquine (HCQ) ${ }^{15}$ additionally improve $\beta$-cell function in patients with RA. HCQ further increases adiponectin production ${ }^{15}$.

In this issue of The Journal, Tejera-Segura and colleagues ${ }^{16}$ report a comprehensive analysis of glucose metabolism in 151 nondiabetic patients with RA and 210 control subjects. The included patients had severe RA; they experienced moderate/uncontrolled disease activity [mean (SD) Disease Activity Score = 3.7 (1.2)] despite the use of prednisone, synthetic conventional DMARD, and biologic agents in $38 \%, 85 \%$, and $23 \%$ of them, respectively. Both circulating insulin and C-peptide concentrations were measured and HOMA2 modeling with optimal use of insulin and C-peptide levels was applied (i.e., insulin sensitivity and insulin secretion were estimated using insulin and C-peptide concentrations, respectively). The relationship of C-peptide production with insulin sensitivity was evaluated. The insulin-C-peptide molar ratio was calculated as a measure of hepatic insulin extraction.

This investigation produced a range of novel findings. First, in univariate analysis, insulin sensitivity as calculated from fasting insulin and glucose concentrations was similar in patients and controls. However, once characteristics that differed between controls and patients were adjusted for, insulin sensitivity was reduced in RA. This indicates that an interaction between RA and other patient characteristics mediated reduced insulin sensitivity. Second, whereas age and diastolic blood pressure were more strongly associated with insulin sensitivity indices in RA than in control subjects, the opposite applied to other traditional insulin resistance risk factors including adiposity, lipids, and current inflammation as estimated by C-reactive protein concentrations. Third, hepatic insulin extraction was increased in RA. Fourth, the relationship between $\beta$-cell function as represented by $\mathrm{C}$-peptide concentrations and insulin sensitivity was weaker in patients with RA compared with controls. These results show that glucose metabolism is consistently different in RA compared with non-RA subjects.

The most striking finding in this study is that for any given insulin sensitivity below 200 according to the HOMA2\% 
insulin sensitivity (which applied to all except for 1 patient with RA and 4 control subjects), C-peptide concentrations were larger in RA compared to control participants. This indicates that the disposition index and hence $\beta$-cell function was actually increased in RA. This finding, together with the identified increased hepatic insulin extraction, are intriguing because they are directly opposite of what is typical of pre-diabetic conditions 7,8 .

Why would insulin secretion be increased to a larger extent than predicted by reduced insulin sensitivity in RA? In this regard, Tejera-Segura and colleagues ${ }^{17}$ previously reported another study on incretin metabolism in the same group of patients with RA and controls that formed part of the current study. Incretins are hormones that are produced by the gut upon food ingestion ${ }^{18}$. They are secreted into the circulation and account for about 50\% of the insulin production upon oral glucose intake. Identified incretins include gastric inhibitory polypeptide (GIP) and glucagon-like peptide-1 (GLP-1). These are both rapidly catabolized by dipeptidyl peptidase 4 (DPP4). The insulinotropic effect of GIP is lost in diabetes. GLP-1 analogs and DPP4 inhibitors are currently used in diabetes.

This study revealed that GIP and GLP-1 concentrations were increased and DPP4 levels were reduced in RA ${ }^{17}$. The erythrocyte sedimentation rate was associated with increased GIP concentrations. Glucocorticoid use was related to increased GIP and GLP-1 and reduced DPP4 levels (as well as enhanced C-peptide concentrations) in RA. Upon adjustment for traditional insulin resistance risk factors, GLP-1 was strongly associated with HOMA2\% insulin secretion in RA but not in control subjects, and the respective relationship differed between both groups (interaction $\mathrm{p}=0.003$ ) Taken together, inflammation and glucocorticoid-mediated increased incretin production and effects could account for the excess insulin production in relation to insulin sensitivity in patients with RA. This phenomenon would represent an overcompensation of $\beta$-cell insulin secretion that may result in its premature decompensation in RA. Further, such overcompensation of $\beta$-cell insulin secretion would be expected to result in increased hepatic insulin extraction ${ }^{8}$, as was also found in the Tejera-Segura study.

Interestingly, American black children similarly experience greater insulin secretion than predicted by insulin resistance ${ }^{19}$. However, in contrast to the findings in the Tejera-Segura study, hepatic insulin extraction is impaired in American black children ${ }^{19}$. The prevalence of diabetes is markedly increased in adult American blacks.

In 2011, Hoes and colleagues ${ }^{20}$ reported on glucose metabolism in patients with RA and control subjects. HOMA modeling and the OGTT were performed. In keeping with the Tejera-Segura study, patients with RA were less insulin-sensitive in the fasting state, but insulin sensitivity was similar in both groups during the OGTT. Overall C-peptide production and hepatic insulin extraction were also increased in RA during the OGTT. However, the disposition index was reduced during the OGTT in RA. This is reminiscent of reported results in other diabetes-predisposing conditions ${ }^{7}$. The discrepancy between the Tejera-Segura and Hoes studies may be due to their difference in RA duration, the mean of which was 7 years in the former and 13 years in the latter ${ }^{16,20}$. This interpretation would again be in keeping with $\beta$-cell overcompensation leading to early decompensation in relation to insulin sensitivity among patients with RA.

Based on reported evidence, we propose that the increased risk of diabetes in RA may be mediated at least in part by systemic inflammation and oral glucocorticoid-induced increased incretin production that causes $\beta$-cell overcompensation in relation to decreased insulin sensitivity and consequent premature decompensation. This hypothesis is further illustrated in Figure 1 and merits future validation.

PATRICK H. DESSEIN (D), MD, FCP (SA), FRCP (UK), PhD, Cardiovascular Pathophysiology and Genomics Research Unit,

School of Physiology,

Faculty of Health Sciences,

University of the Witwatersrand, and Department of Rheumatology,

Charlotte Maxeke Johannesburg Academic Hospital,

Faculty of Health Sciences,

University of the Witwatersrand,

and Free University and University Hospital,

Brussels, Belgium;

ANNE E. STANWIX, MBвCH, FRCP,

Department of Rheumatology,

Charlotte Maxeke Johannesburg Academic Hospital,

Faculty of Health Sciences,

University of the Witwatersrand;

AHMED SOLOMON, MBBCH, FCP (SA), PhD,

Department of Rheumatology,

Charlotte Maxeke Johannesburg Academic Hospital,

Faculty of Health Sciences,

University of the Witwatersrand,

Johannesburg, South Africa.

Address correspondence to Dr. P.H. Dessein, Cardiovascular Pathophysiology and Genomics Research Unit, School of Physiology, University of the Witwatersrand Medical School, 7 York Road, Parktown, 2193, Johannesburg, South Africa. E-mail: patrick.dessein22@gmail.com The work was supported by the South African National Research Foundation and Medical Research Council.

\section{REFERENCES}

1. Dessein PH, Solomon A, Hollan I. Metabolic abnormalities in patients with inflammatory rheumatic diseases. Best Pract Res Clin Rheumatol 2016;30:901-15.

2. Bayer JF, Gourraud PA, Cantagrel A, Davignon JL, Constantin A Traditional cardiovascular risk factors in rheumatoid arthritis: a meta-analysis. Joint Bone Spine 2011;78:179-83.

3. Baghdadi LR, Woodman RJ, Shanahan EM, Mangoni A. The impact of traditional cardiovascular risk factors on cardiovascular outcomes in patients with rheumatoid arthritis: a systematic review and meta-analysis. PLoS One 2015;10:e0117952. 
4. Ruscitti P, Ursini F, Cipriani P, Liakouli V, Carubbi F, Berardicurti $\mathrm{O}$, et al. Poor clinical response in rheumatoid arthritis is the main risk factor for diabetes development in the short-term: A 1-year single-centre, longitudinal study. PLoS One 2017;12:e0181203.

5. Lewis GF, Carpentier A, Adeli K, Giacca A. Disordered fat storage and mobilization in the pathogenesis of insulin resistance and type 2 diabetes. Endocrine Reviews 2002;23:201-29.

6. Dessein PH, Joffe BI, Stanwix AE. Inflammation, insulin resistance, and aberrant lipid metabolism as cardiovascular risk factors in rheumatoid arthritis. J Rheumatol 2003;30:1403-5.

7. Kahn SE, Prigeon RL, Schwarts RS, Fujimoto WY, Knopp RH, Brunzell JD, et al. Obesity, body fat distribution, insulin sensitivity and islet beta-cell function as explanations for metabolic diversity. J Nutr 2001;131:354S-360S.

8. Meier JJ, Veldhuis JD, Butler PC. Pulsatile insulin secretion dictates systemic insulin delivery by regulating hepatic insulin extraction in humans. Diabetes 2005;54:1649-58.

9. Cersosimo E, Solis-Herrera C, Trautman ME, Malloy J, Triplitt CL. Assessment of pancreatic $\beta$-cell function: review of methods and clinical applications. Curr Diabetes Rev 2014;10:2-42.

10. Nicolau J, Lequerre T, Bacquet H, Vittecoq O. Rheumatoid arthritis, insulin resistance, and diabetes. Joint Spine Bone 2017;84:411-6.

11. Dessein PH, Joffe BI. Insulin resistance and impaired beta cell function in rheumatoid arthritis. Arthritis Rheum 2006;54:2765-75

12. Dessein PH, Joffe BI, Stanwix AE. Subclinical hypothyroidism is associated with insulin resistance in rheumatoid arthritis. Thyroid 2004;14:443-6

13. Ferraz-Amaro I, Garcia-Dopico JA, Medina-Vega L, Gonzalez-Gay MA, Diaz-Gonzalez F. Impaired beta cell function is present in nondiabetic rheumatoid arthritis patients. Arthritis Res Ther 2013;15:R17.
14. Stagakis I, Bersias G, Karvounaris S, Kavousanaki M, Virla D, Raptopoulou A, et al. Anti-tumor necrosis factor therapy improves insulin resistance, beta cell function and insulin signaling in active rheumatoid arthritis patients with high insulin resistance. Arthritis Res Ther 2012;14:R141.

15. Wasko MC, McClure CK, Kelsey SF, Huber K, Orchard T, Toledo FG. Antidiabetogenic effects of hydroxychloroquine on insulin sensitivity and beta cell function: a randomised trial. Diabetologia 2015;58:2336-43.

16. Tejera-Segura B, Lopez-Mejias R, de Vera-Gonzalez AM, Jiménez-Sosa A, Olmos JM, Hernández JL, et al. Relationship between insulin sensitivity and $\beta$-cell secretion in nondiabetic subjects with rheumatoid arthritis. J Rheumatol 2019;46:229-36

17. Tejera-Segura B, López-Mejías R, Dominguez-Luis MJ, de Vera-González AM, González-Delgado A, Ubilla B, et al. Incretins in patients with rheumatoid arthritis. Arthritis Res Ther 2017;19:229.

18. Kim W, Egan JM. The role of incretins in glucose homeostasis and diabetes treatment. Pharmacol Rev 2008;60:470-512.

19. Gower BA, Granger WM, Franklin F, Shechuk RM, Goran MI. Contribution of insulin secretion and clearance to glucose-induced insulin concentrations in African-American and Caucasian children. J Clin Endocrinol Metab 2002;87:2218-24.

20. Hoes JN, van der Goes MC, van Raalte DH, van der Zijl NJ, den Uyl D, Lems WF, et al. Glucose tolerance, insulin sensitivity and $\beta$-cell function in patients with rheumatoid arthritis treated with or without low-to-medium dose glucocorticoids. Ann Rheum Dis 2011;70:1887-94.

J Rheumatol 2019;46:219-22; doi:10.3899/jrheum.181163 